\title{
Desarrollo metodológico para medir el trabajo en equipo
}

\author{
Viles, Elisabeth* \\ Jaca, Carmen** \\ Tanco, Martín*** \\ Medina, Allan****
}

\section{Resumen}

En el sector sanitario, el trabajo en equipo es una parte importante de su actividad. Aunque el sector fomenta la investigación orientada a mejorar la calidad del trabajo en equipo, pocas veces se focaliza a la evaluación de este proceso. Por esta razón en el presente artículo se desarrolla una propuesta metodológica para evaluar la efectividad del trabajo en equipo. Para ello el modelo contempla 36 factores que son utilizados para su medición, a través de la observación de la existencia, importancia y nivel de aplicación de éstos en el trabajo de equipo. El modelo fue utilizado en la evaluación de equipos de trabajo de la red pública de hospitales en el País Vasco (España), la metodología utilizada ha sido del tipo exploratorio, y se desarrolló con los datos obtenidos a partir de entrevistas realizadas siguiendo un guión basado en la estructura del propio modelo. De la aplicación del modelo al caso práctico se puede concluir que la mayoría de los factores analizados en estos hospitales obtuvieron puntuaciones medias y altas, siendo las más bajas las relacionadas con los resultados, el reconocimiento, la formación y la selección de los miembros de los equipos.

Palabras clave: Equipos de trabajo, efectividad, caso hospitalario, evaluación cualitativa.

Recibido: 20-10-10. Aceptado: 25-05-11

* Doctora en Ciencias. Profesora titular del Departamento de Organización de Empresas. TECNUN. Campus Tecnológico de la Universidad de Navarra. Paseo Manuel Lardizábal, 13, 20018 San Sebastián, España. E-mail: eviles@tecnun.es

** Ingeniera Industrial. Profesora del Departamento de Organización Industrial. TECNUN, Campus Tecnológico de la Universidad de Navarra. Paseo Manuel Lardizábal, 13, 20018 San Sebastián, España. E-mail: cjaca@tecnun.es

*** Doctor en Ingeniería Industrial. Profesor de la Universidad de Montevideo. Luis P. Ponce 1307,11300 Montevideo, Uruguay. E-mail: mtanco@um.edu.uy / mtanco@um.edu.uy

**** Ingeniero Industrial. Supermercados La Colonia. Departamento de Logística. Col. Alameda Calle Rubén Darío, Contiguo a Droguería Nacional. Apdo Postal 3225. Tegucigalpa M.D.C, Honduras CA. E-mail: amedina@lacolonia.hn 


\title{
Methodological Development for Measuring Teamwork. Application at Hospitals in the Basque Country Public Health Network (Osakidetza)
}

\begin{abstract}
In the healthcare sector, teamwork is a key issue. Although the sector promotes research on improving the quality of teamwork, it rarely focuses on evaluating this process. This paper develops a methodological proposal for assessing teamwork effectiveness. The model considers 36 factors used to measure it, by observing the existence, importance and application level of these factors in the teamwork. The model was used to assess teams working in the public hospital network in the Basque Country (Spain). Methodology was of the exploratory type and developed with data obtained from interviews carried out according to a script based on the model's structure. The assessment was conducted through interviews in order to qualitatively assess the existence, degree and level of implementation for those factors. From applying the model to the practical case, conclusions are that most of the factors analyzed in those hospitals had medium and high scores; the lowest scores were for factors related to results, recognition, training and selecting team members.
\end{abstract}

Key words: Work teams, effectiveness, hospital case, qualitative evaluation.

\section{Introducción}

El trabajo en equipo representa para las organizaciones una herramienta poderosa para dar respuesta a las crecientes exigencias externas (productividad, competitividad y tecnología) e internas (trabajadores motivados, productivos y participativos) (Mantilla y García, 2010), y es la base fundamental de cualquier metodología de mejora continua.

El sector sanitario destaca entre otros sectores en el desarrollo e investigación del trabajo en equipo, en este sector el trabajo en equipo se considera parte natural del trabajo habitual de médicos, enfermeras, técnicos de laboratorio, auxiliares y resto de personal sanitario.

En el sector hospitalario, el trabajo en equipo beneficia a los miembros del equipo, a la organización y a los pacientes (Xyrichis y Ream, 2008). Los miem- bros están más satisfechos con su trabajo y más motivados ya que se reconoce la aportación individual de cada miembro al equipo (Borrill et al., 2000). La organización se beneficia al tener una plantilla más satisfecha y comprometida, una menor rotación y un mayor control de los costes. Y los pacientes se benefician de una mejor atención y una mayor satisfacción con los servicios (Bower et al., 2003).

Aunque el sector fomenta la investigación orientada a mejorar la calidad del trabajo en equipo, pocas veces se cuestiona que el proceso de trabajar en equipo también debiera ser evaluado. De hecho, existen pocos estudios en los que se analice el proceso del trabajo en equipo en casos reales (Mathieu et al., 2008) y los existentes no aportan una herramienta para su evaluación. Por tanto el objetivo de este artículo consiste en desarrollar una propuesta metodológica para eva- 
luar la efectividad del trabajo en equipo, considerando factores que distintos autores han apuntado como determinantes para la eficiencia del trabajo en equipo. La propuesta metodológica que se aporta ha sido aplicada posteriormente para la evaluación de distintos equipos de trabajo hospitalarios.

Como punto de partida en este estudio, ha sido necesario consensuar el concepto y la definición de trabajo en equipo. En la bibliografía analizada tanto la relacionada con la gestión de las organizaciones como en la propia del sector sanitario, se pueden encontrar varias y variadas definiciones del trabajo en equipo (Hackman, 1990, Guzzo y Dickinson, 1996, Katzenbach y Smith, 1993, Salas et al., 2005, Zander, 1994). La definición de equipo utilizada en este estudio ha sido la siguiente:

Se llama equipo de trabajo a un pequeño grupo de personas con habilidades y conocimientos complementarios que están comprometidos con una misión, un conjunto de metas de desempeño y con un enfoque común para los cuales son mutuamente responsables (Katzenbach y Smith, 1993).

Por otro lado, la estructura del equipo difiere dependiendo de su propósito, su tarea, su entorno, su composición y las relaciones formales entre los profesionales del equipo (Sundstrom et al., 2000). En el sector sanitario existen varios tipos de equipos. Los más comunes en el sector sanitario son (Oandasan et al., 2006):

a) Equipos de proyecto: formados específicamente para resolver un problema o realizar una tarea en particular. Por ejemplo: equipos de mejora de calidad. b) Equipos de gestión: formados para dirigir a la organización y tomar las decisiones que tendrán un mayor impacto en la organización. Los equipos de dirección son un ejemplo típico.

c) Equipos de asistencia médica: formados para brindar asistencia médica. Estos equipos pueden variar según el área, la enfermedad y el paciente.

Este estudio se ha centrado en los equipos de asistencia médica de carácter permanente. Dentro de los distintos tipos de equipos en el sector médico, éstos son los más dinámicos y complejos, debido a que frecuentemente los miembros y el equipo se encargan de diagnosticar, resolver problemas y/o colaborar estrechamente para acometer la tarea asignada en cada momento (Brannick et al., 1997). Estos equipos aprovechan los conocimientos y habilidades adquiridas a través de la experiencia para ofrecer cada vez servicios más eficientes.

En este artículo se analizan cómo es la aplicación de los factores que afectan al trabajo en equipo en los equipos asistenciales de los hospitales de la red pública vasca (Osakidetza). Para ello, se ha tomado como referencia el modelo de trabajo en equipo adaptado y desarrollado por Mathieu et al., (2008), el cual se ha completado con la identificación de los factores clave en cada nivel del modelo. Partiendo de este modelo teórico se ha evaluado su aplicación, con el fin de obtener conclusiones acerca del funcionamiento del trabajo en equipo. Finalmente se propone este modelo adaptado y completado, como herramienta para evaluar distintos equipos en instituciones médicas. 
Desarrollo metodológico para medir el trabajo en equipo

Viles, Elisabeth; Jaca, Carmen; Tanco, Martín; Medina, Allan

\section{Modelo para medir la efectividad del trabajo en equipo}

A continuación se expone el modelo utilizado en el artículo, con una breve explicación de la metodología utilizada para seleccionar los factores de dicho modelo y para la posterior aplicación del mismo.

El modelo utilizado en esta investigación es el modelo integrado de efectividad de trabajo en equipo, IMO, desarrollado por Ilgen (2005) y Mathieu (2008), que es un modelo que se ajusta a los equipos de trabajo asistencial del sector sanitario. El modelo IMO, representa el carácter cíclico de los procesos de retroalimentación que ocurren en el equipo, de forma que los resultados (output) se convierten en nuevos input para el proceso
(Kozlowski y llgen, 2006). Este modelo considera a los equipos como un sistema multinivel orientado a los procesos relevantes para el objetivo del equipo, y que evoluciona en el tiempo, de manera que tanto los procesos como la eficiencia del equipo influyen en el desarrollo del equipo y trabajo (Rico et al., 2010). Este modelo se representa en el Diagrama 1.

En este modelo se agrupan los factores que afectan al equipo en tres grandes grupos: inputs, mediators y outcomes (Modelo IMO).

En los inputs se agrupan los factores previos a la formación del equipo. Estos factores pueden clasificarse en tres niveles: miembros, equipo o contexto organizativo. En el modelo se observa que los tres grupos están anidados. De esta forma se representa la fuerte influencia

\section{Diagrama 1 \\ Modelo IMO (Input- Mediators-Outcome) del desarrollo del trabajo en equipo}
INPUT
MEDIATORS
OUTCOMES

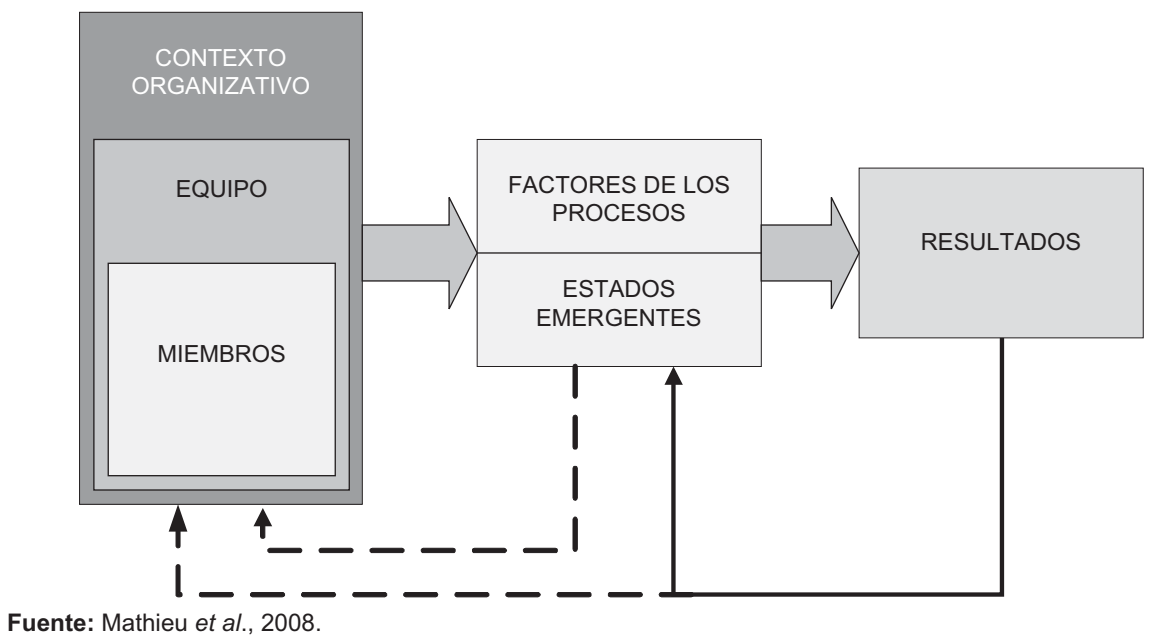


que tienen los factores del contexto organizativo sobre el equipo y, a su vez, cómo el equipo influye sobre los miembros exigiéndoles ciertas competencias y distribución de roles.

- El contexto organizativo hace referencia a todos aquellos factores que existen dentro de la organización y que influyen en el diseño de los equipos y en el entorno de trabajo.

- Los factores pertenecientes al equipo son aquellos que están relacionados con el funcionamiento del propio equipo, que están definidos o existen antes de que el equipo empiece a trabajar como tal.

- Los factores pertenecientes a los miembros incluyen las características individuales de los miembros del equipo.

En los mediators se incluyen los factores que influyen durante el proceso del trabajo en equipo. Algunos de estos factores suponen acciones de los miembros mientras que otros se consideran como estados afectivos, cognitivos o referentes a la motivación (Marks et al., 2001). Es por ello que los mediators se dividen en factores de procesos y en estados emergentes. Los factores de los Procesos describen funciones e interacciones que aparecen durante el trabajo en equipo y cómo son gestionadas por la organización, el equipo y los miembros. Los Estados Emergentes son factores dinámicos que aparecen durante el proceso del trabajo en equipo y que varían en el tiempo dependiendo del resto de los factores, Input, Procesos y Outputs (Mathieu et al., 2008).

Por último, en los outcomes se agrupan los factores relacionados con el de- sempeño del trabajo en equipo y, por tanto, se valoran los resultados tanto del equipo como de los miembros y de la organización.

En el modelo se representa mediante bucles de retroalimentación la característica cíclica de los procesos realizados por los equipos. Las líneas sólidas de retroalimentación sugieren que los resultados influyen de manera importante en los procesos y estados emergentes. Las líneas punteadas indican que la influencia sobre los inputs es menor al ser más difícil de cambiar la estructura y composición del equipo así como el contexto organizativo.

\subsection{Factores asociados al modelo}

Para los autores de este artículo, disponer de una manera de evaluar el modelo anterior resulta un valor añadido al propio modelo, ya que establece una referencia para la mejora de los equipos de trabajo. Y para poder medir la eficacia del trabajo en equipo, es necesario previamente identificar factores que puedan ser valorados en cada uno de los grupos contenidos dentro de los inputs, mediators y outcomes del modelo.

En la actualidad existen pocos estudios donde se analicen modelos IMO y a la vez se evalúen los factores que caracterizan el buen desempeño de los equipos de trabajo (Louise LemieuxCharles, Michael Murray, G. Ross Baker, Jan Barnsley, Kevin Tasa,Salahadin A.Ibrahim, 2002, Mendibil y MacBryde, 2005, Mickan y Rodger, 2005). Y éste ha sido uno de los objetivos del trabajo que aquí se presenta: identificar y ligar los factores a las distintas partes 
Desarrollo metodológico para medir el trabajo en equipo

Viles, Elisabeth; Jaca, Carmen; Tanco, Martín; Medina, Allan

del modelo, para posteriormente poder ser evaluados.

Para la identificación y definición de los factores que se presentan en este trabajo, se ha combinado el conocimiento de los autores con el estudio de distintas fuentes bibliográficas (Mathieu et al., 2008, Cohen y Bailey, 1997, Ilgen et al, 2005, McGrath et al., 2000, Mickan y Rodger, 2000, Lemieux-Charles y McGuire, 2006, Thylefors et al, 2005). En el Diagrama 2 se listan todos los factores que se han identificado en el modelo organizados de acuerdo a los inputs, mediators y outcomes.
A partir de la aceptación de este modelo como modelo conceptual que representa la manera en la que debiera transcurrir con eficacia el trabajo en equipo, se ha diseñado una encuesta para evaluar en qué medida los factores descritos tienen lugar.

\subsection{Metodología para la evaluación del modelo}

La metodología utilizada para tal fin ha sido del tipo exploratorio, y ha sido realizada con los datos obtenidos a partir de entrevistas realizadas siguiendo un guión

\section{Diagrama 2 \\ Modelo IMO para medir la efectividad del trabajo en equipo con los factores identificados \\ INPUT \\ MEDIATORS \\ OUTCOMES}

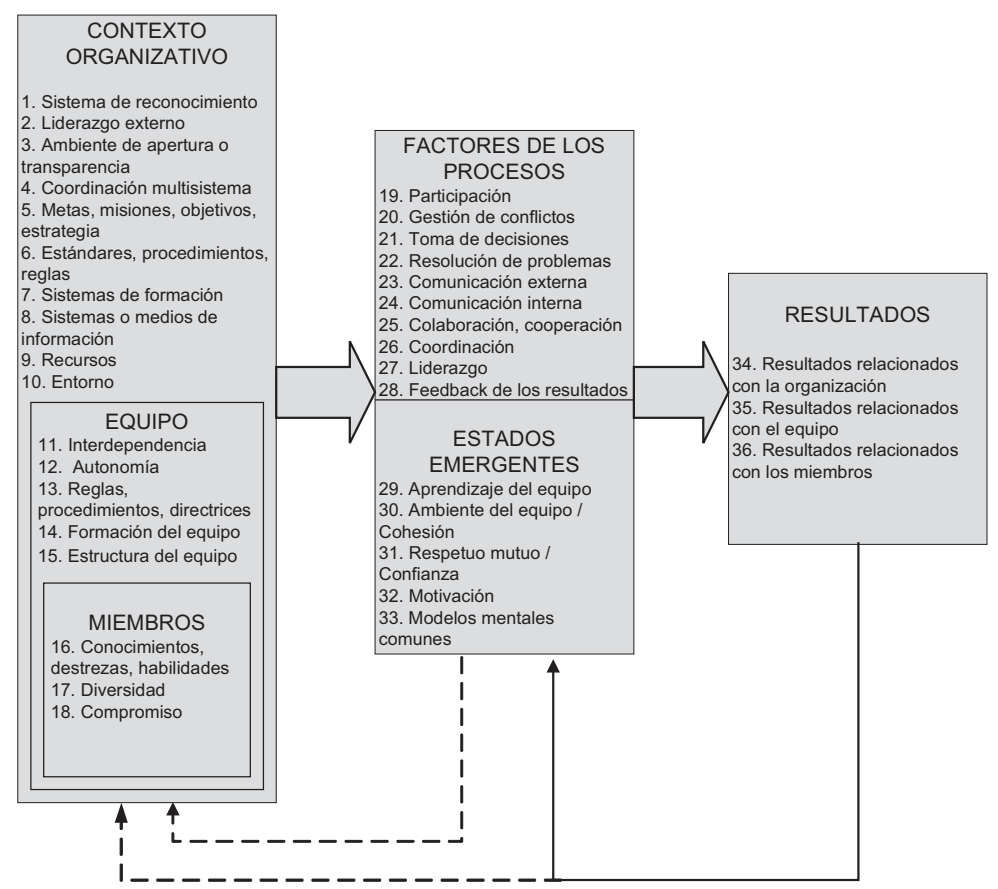

Fuente: Elaboración propia. 
(de creación propia) basado en la estructura del propio modelo. Esta técnica de investigación es muy apropiada cuando el objeto de la exploración es el estudio o la evaluación de un proceso (Patton, 2002).

El guión confeccionado para tal fin se creó definiendo cada uno de los factores para evitar confusiones y para facilitar la comunicación durante el desarrollo de la entrevista. Cada factor se desplegó en 5 niveles, según su nivel de aplicación, medición y evaluación, siguiendo el siguiente esquema:

1) No se considera el factor $X$ en el trabajo en equipo.

2) El factor $X$ se considera importante para el trabajo en equipo.

3) El factor $X$ se aplica en los equipos.

4) Se tiene en cuenta el factor $X$, se considera importante, y además se aplica muy frecuentemente o siempre.

5) Se tiene en cuenta el factor $X$, se considera importante, se aplica siempre y además existen registros y/o indicadores asociados a su aplicación.

La adecuada definición de los factores fue fundamental para identificar la importancia y aplicación que a cada factor se le da dentro del equipo. Por este motivo, se decidió establecer por escrito una definición clara de cada factor que evitara confusiones y facilitara la comunicación durante el desarrollo de la evaluación.

Inicialmente se realizó una entrevista piloto con un médico perteneciente a uno de los grupos a analizar. Esta prueba permitió realizar cambios y mejoras en la metodología de las entrevistas que permitieron establecer el correcto dimensionamiento de la misma (en tiempo y tamaño), obligaron a establecer una terminología común entre las partes y, por tanto, fa- cilitaron la recogida posterior de la información.

Una vez validado el guión de la entrevista, se decidió llevarla a cabo de manera personalizada con el objetivo de obtener como resultado la ponderación del modelo y detectar además la razón de determinadas actuaciones. De esta forma pudieron recogerse evidencias de buenas prácticas y áreas de mejora para algunos de los factores analizados. La totalidad de las entrevistas se mantuvieron con los jefes de cada servicio, que son los que con mayor probabilidad tienen la visión práctica del servicio y la de su gestión.

El área geográfica elegida para la investigación ha sido la Comunidad Autónoma Vasca (CAV), situada en el norte de España. A pesar de que su extensión geográfica no es muy grande, la gestión de las organizaciones vascas, y en concreto las organizaciones de la red pública sanitaria, es reconocida en toda Europa por su calidad y prestigio.

La Comunidad Autónoma Vasca fue la región más premiada en Europa por la Fundación Europea de la Calidad (EFQM) en el periodo comprendido entre 2000 y 2010. 23 de los Premios Europeos de Calidad, incluyendo finalistas, premiados y galardonados, han recaído en organizaciones pertenecientes a esta región. La EFQM es una organización sin ánimo de lucro que trabaja para promover la mejora de la calidad y de la gestión. Una de sus actividades es el reconocimiento de las empresas que han obtenido un elevado nivel de mejora en su gestión, utilizando como criterio el Modelo de Excelencia EFQM. Este modelo, introducido en 1991, es similar al Malcom Baldridge o al Modelo Iberoamericano de Excelencia en la gestión. En la Comunidad 
Desarrollo metodológico para medir el trabajo en equipo

Viles, Elisabeth; Jaca, Carmen; Tanco, Martín; Medina, Allan

Autónoma Vasca la organización Euskalit trabaja a su vez para promover localmente la cultura de la Calidad Total. Esta entidad otorga un reconocimiento llamado $Q$ de Oro o $Q$ de plata a las organizaciones que consigan más de 400 puntos en su evaluación EFQM.

Los hospitales seleccionados para el estudio han sido hospitales que en los últimos años han recibido este galardón a la excelencia de su gestión. Por otra parte, se decidió focalizar el estudio sobre cuatro actividades del hospital cuya operativa se basara fundamentalmente en el trabajo en equipo de tipo asistencial. Bajo estas premisas se evaluaron las unidades de a) cuidados intensivos, b) el servicio de urgencias, c) el quirófano de traumatología y d) la planta de cardiología. Los hospitales finalmente seleccionados y accesibles fueron 5, obteniéndose un total de 17 entrevistas. Estas entrevistas fueron realizadas durante los meses de Junio y Julio de 2009.

\section{Modelo para medir la efectividad del trabajo en equipo: caso práctico}

A continuación se muestra en el Diagrama 3, los valores medios obtenidos para cada conjunto de factores dentro de los inputs, mediators y outcomes.

Las gráficas de la Figura 1 muestran los valores medios obtenidos por los factores al ser agrupados por área de servicio. Se incluyen también los datos globales para poder observar las desviaciones existentes entre las áreas y los datos globales del estudio.

\section{Diagrama 3 \\ Modelo IMO de la efectividad del trabajo en equipo con las puntuaciones medias obtenidas}
INPUT
MEDIATORS
OUTCOMES

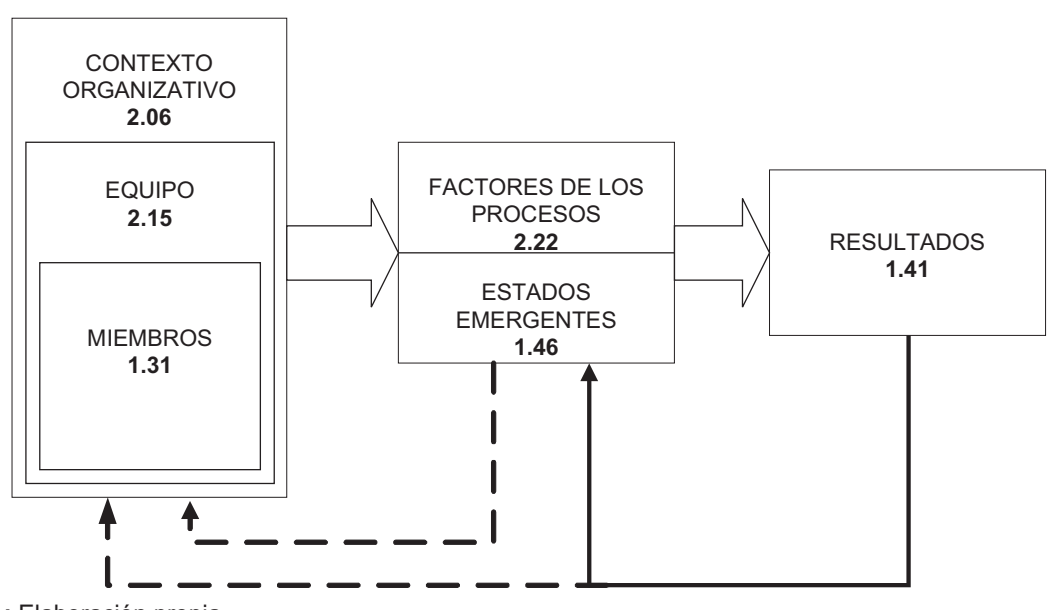

Fuente: Elaboración propia. 


\section{Figura 1}

Valores medios obtenidos por los factores al ser agrupados por área de servicio.
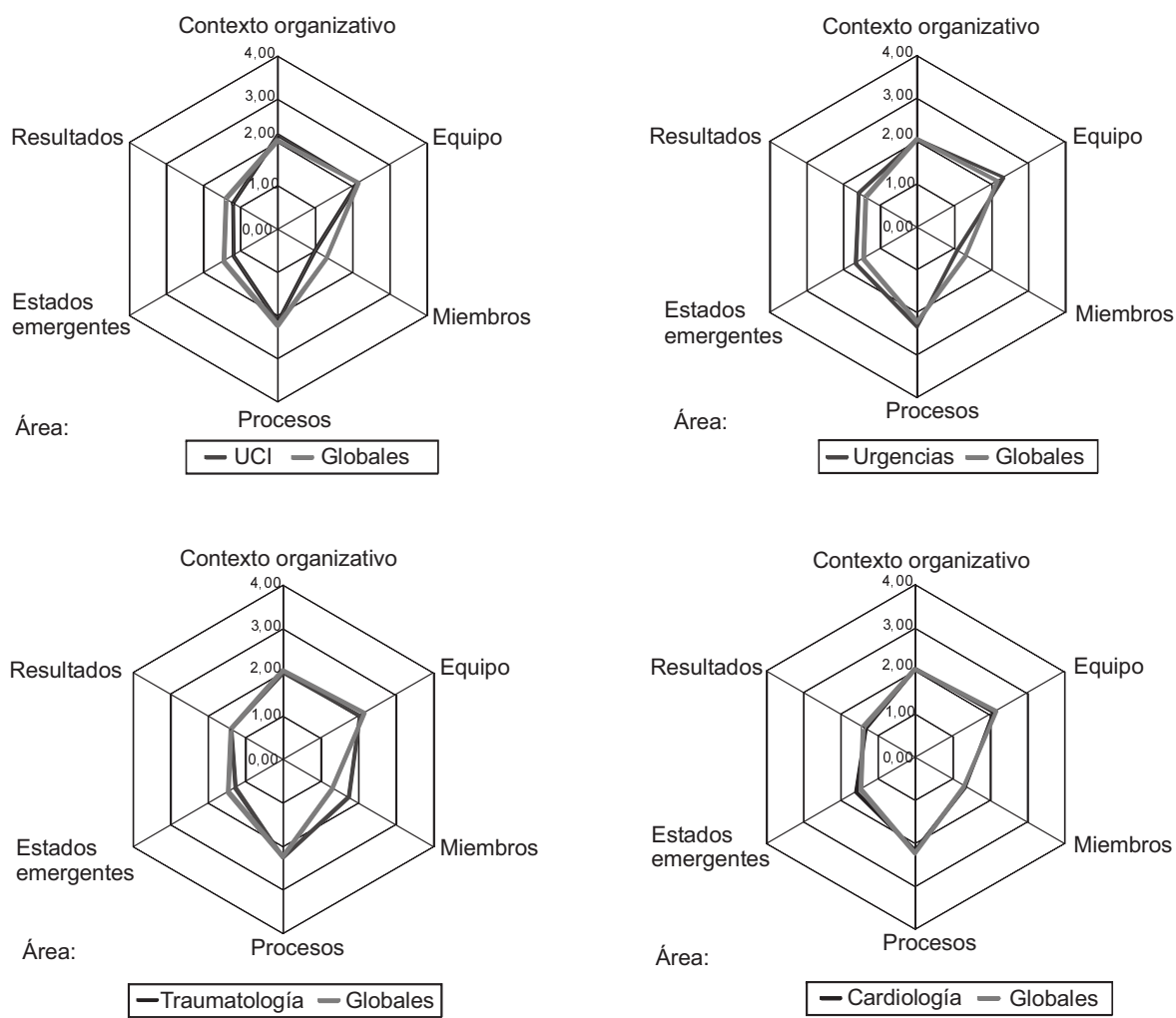

Fuente: Elaboración propia.

Como puede verse en la Figura 1, las medias de los factores por servicio presentan pequeñas diferencias respecto a la media total de cada categoría de factores, lo que refuerza la coherencia de las entrevistas. La diferencia más significativa es la del área de traumatología en el grupo miembros. Al ser el quirófano de

trauma un equipo formado por cirujanos y profesionales altamente cualificados la selección y composición de los equipos se considera muy importante. Las Gráficas 1,2 y 3 , detallan los factores para los cuales se han obtenido valores más extremos y su variabilidad respecto del área analizada. 
Desarrollo metodológico para medir el trabajo en equipo

Viles, Elisabeth; Jaca, Carmen; Tanco, Martín; Medina, Allan

\section{Gráfica 1}

Valores obtenidos por los factores analizados en el INPUT del modelo IMO relacionados con el contexto organizativo, agrupados por área de servicio.

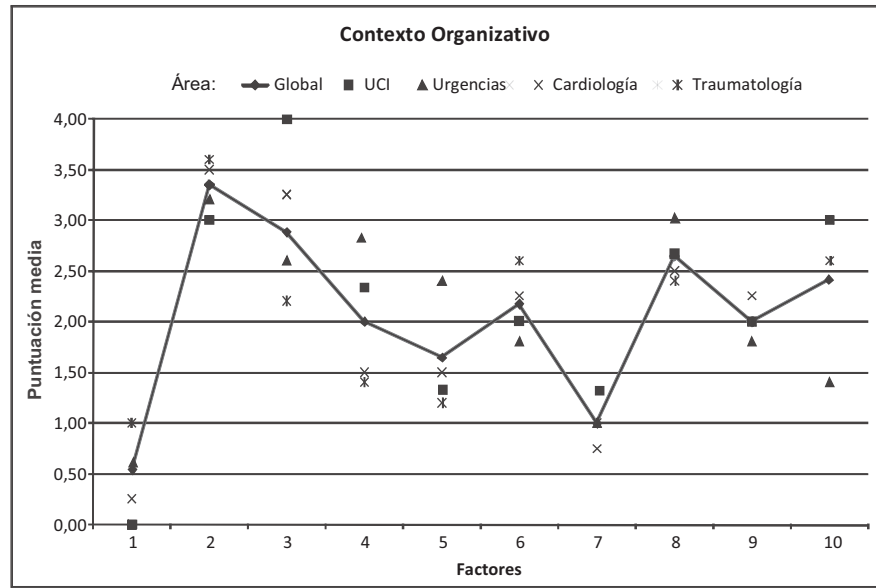

Factores:

1. Reconocimiento

2. Liderazgo externo

3. Ambiente de apertura y

transparencia

4. Coordinación

multisistema

5. Metas, misión, objetivos

y estrategia

6. Estándares,

procedimientos, reglas

. Sistemas de formación

(técnica)

8. Sistemas o medios de

información

9. Recursos

10. Entorno

Fuente: Elaboración propia.

Gráfica 2

Valores obtenidos por los factores analizados en el INPUT del modelo IMO relacionados con los equipos y sus miembros y agrupados por área de servicio.

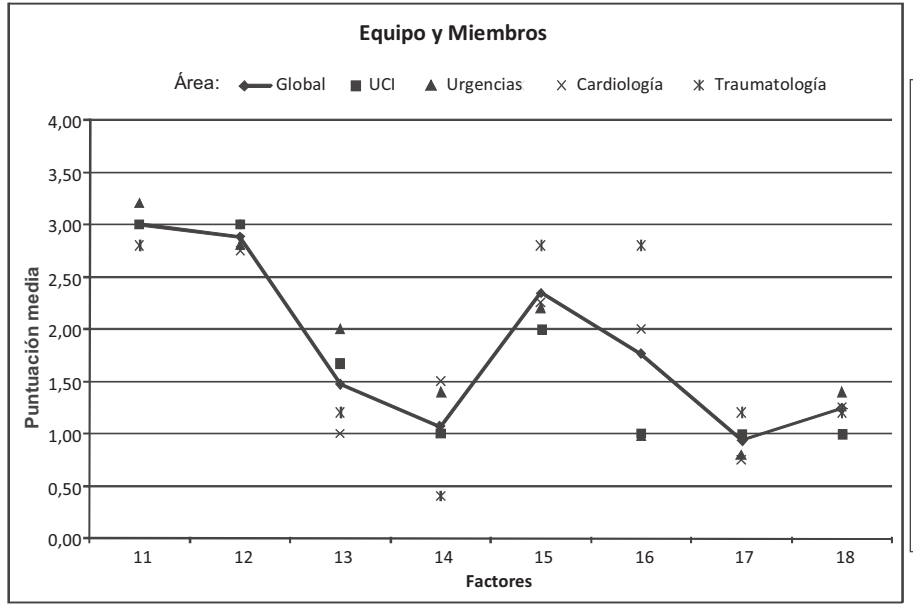

Factores:

11. Interdependencia

12. Autonomía

13. Reglas,

procedimientos,

directrices

14. Formación del

equipo (conjunto)

15. Estructura del

equipo

16. Conocimientos,

destrezas, actitudes

17. Diversidad

18. Compromiso

Fuente: Elaboración propia. 


\section{Gráfica 3 \\ Valores obtenidos por los factores asociados a OUTCOMES del modelo IMO}

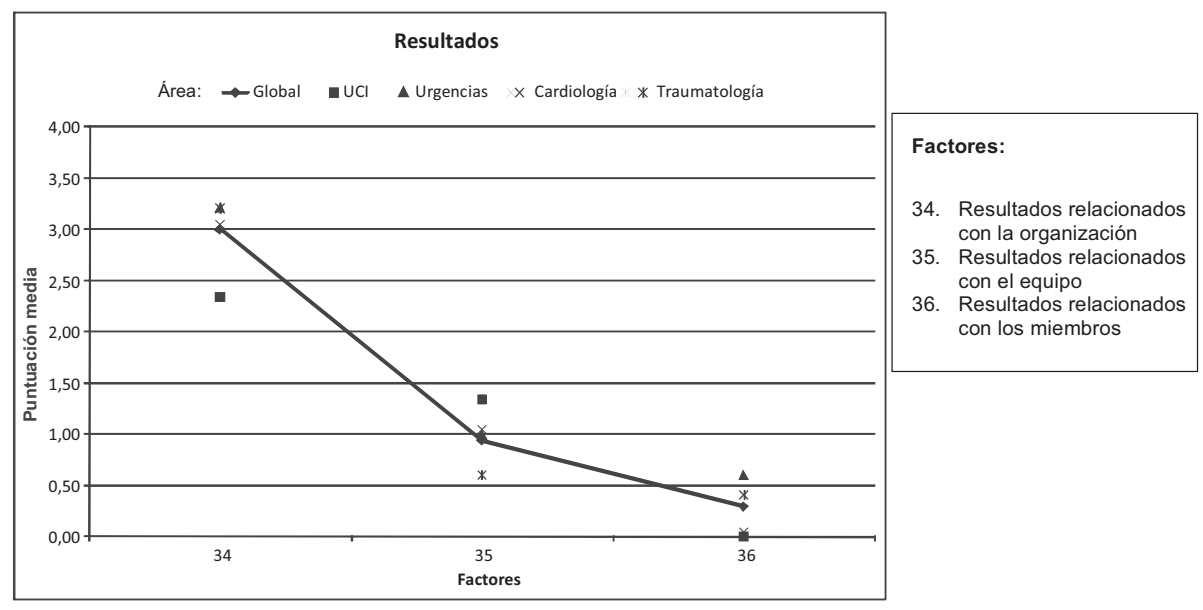

Fuente: Elaboración propia.

Dentro del contexto organizativo, son 4 los factores que presentan más desviaciones (factores 1, 3, 4, 5 y 10).

En cuanto al reconocimiento (factor 1), a pesar del descontento expresado por la mayoría de los médicos con la implantación de la carrera profesional, algunas áreas del hospital contaban con un sistema de reconocimiento propio. En ellos, los médicos intentaban premiar con formación al personal que más destacaba en el servicio o con días de vacaciones a todo el área. Por ejemplo, en el área de traumatología de uno de los hospitales el sistema de reconocimiento evalúa los resultados obtenidos y si se alcanzan los objetivos se premia al personal con dos o tres días adicionales de vacaciones. $\mathrm{La} \mathrm{UCl}$ tiene la puntuación más baja en reconocimiento en parte debido a que el área de $\mathrm{UCI}$ no está presente en todos los hospitales.
El ambiente de apertura y trasparencia (factor 3) frecuentemente está asociado a la gestión de errores dentro del hospital (Firth-Cozens, 2004). Se ha observado que la gestión de errores no estaba implantada de la misma manera en todas las áreas de los hospitales. La unidad de cuidados intensivos es el área que mayor puntuación recibió debido a su concienciación en la importancia del registro de errores e incidentes y en el posterior análisis de los datos. Como muestra de ello, actualmente la $\mathrm{UCI}$ de otro de los hospitales está inmersa en una dinámica de concienciación de errores, incidentes y efectos adversos. Tienen un sistema implantado donde se registran todos los errores y analizan los datos obtenidos. Una de las mayores dificultades ante las que se encuentran en la utilización diaria del sistema es en la definición de qué es un error y en su clasificación. 
Desarrollo metodológico para medir el trabajo en equipo

Viles, Elisabeth; Jaca, Carmen; Tanco, Martín; Medina, Allan

Mientras algunos hospitales fomentan un ambiente de reporte de todos los errores e incidentes, otros se centran únicamente en los errores médicos, analizados de manera anónima.

El área de urgencias (factor 4) es la que más destaca en la coordinación multisistema ya que sólo en los casos de urgencias las distintas áreas se coordinan para trabajar en conjunto. En todos los hospitales analizados, dentro del área de urgencias se han desarrollado protocolos para facilitar el trabajo en conjunto de las distintas áreas con urgencias. En algunos casos, la organización promueve los grupos inter-disciplinares y hay un ambiente de cooperación y servicio entre las áreas.

El área de urgencias destaca del resto en cuanto a la definición de metas, misiones y objetivos (factor 5). En algunos hospitales, aunque no en todos, la organización plantea objetivos cuantificables al servicio de urgencias que son comunicados a los equipos de trabajo. Posteriormente se obtienen resultados correspondientes a estos objetivos que son analizados globalmente para todo el servicio de urgencias.

El entorno externo e interno (factor 10) afecta especialmente al área de urgencias. El entorno político genera cambios a largo plazo mientras que los cambios sociales y económicos influyen a más corto plazo. Las variables sociales del entorno pueden producir variaciones importantes en la demanda que influye directamente en su ambiente laboral.

Referente a los equipos y miembros, se ha observado que los equipos médicos muestran un alto grado de interdependencia y autonomía (factores $11 \mathrm{y}$
12), con valores medios similares en todas las áreas. Esto es debido a que la organización da libertad para tomar las decisiones relacionadas con el ámbito de relación de los equipos, que por otro lado, requieren un alto grado de interdependencia para la ejecución de sus tareas. En cuanto a la formación en equipo (factor 14), se presentan diferencias entre las áreas. En la práctica, y dependiendo de la cultura del servicio, la formación se recibe de manera individual o, con alguna frecuencia, a dos de los miembros del equipo. No se suele realizar formación conjunta. En cuanto al factor de conocimientos, destrezas y actitudes (factor 16) para la elección de los miembros, este factor es el que único que tiene una desviación importante. Traumatología es el que tiene la puntuación más elevada ya que consideran los conocimientos, destrezas y en algunos casos las actitudes para la elección de los miembros.

En el área de traumatología de uno de los hospitales se le da una especial importancia al hecho de que los equipos sean fijos para poder tener en cuenta los conocimientos, destrezas y compromiso en la elección de los miembros. De esta forma, garantizan que la experiencia de los miembros del equipo sea la adecuada. Para ellos es la clave de la alta productividad que tienen.

En los resultados no se encuentran grandes variaciones entre las áreas. Casi todas las áreas obtienen información relativa a la organización pero muy pocas analizan los resultados de los equipos y ninguno obtiene resultados de los miembros ya que disponen de muy pocos datos para ello. 


\section{Conclusiones}

Tal y como se ha expuesto a lo largo de este artículo, Se ha identificado en la bibliografía un modelo que representa los procesos que deben darse lugar para que los equipos trabajen eficazmente. Partiendo de este modelo y tras una revisión bibliográfica apropiada, se han definido 36 factores que han sido analizados dentro de este modelo para que pueda servir además para medir dicha efectividad.

Para su testeo y validación se decidió aplicarlo al sector hospitalario. En concreto fueron evaluadas 4 unidades de servicio de hospitales con calidad reconocida internacionalmente.

Las conclusiones que directamente se derivan de este análisis pueden resumirse en:

- La mayoría de los factores obtuvieron puntuaciones medias y altas, siendo las más bajas las relacionadas con los resultados, el reconocimiento y la formación.

- El entorno social afecta directamente al trabajo de los equipos. En concreto en este estudio, se constató que la actual escasez de personal hospitalario causó en general que los factores relacionados con la selección de los miembros de los equipos tuvieran puntuaciones medias y bajas.

- El Jefe de servicio en cualquier unidad de un hospital desarrolla un rol importante para los equipos. Es el líder del equipo en la mayoría de los casos. Es responsable del equipo ante los directivos y equipos externos y facilita su coordinación con el resto del sistema.
- El proceso de toma de decisiones y la gestión de conflictos se realiza solamente por el líder (el jefe de servicio). Esto proporciona al equipo la rapidez de respuesta necesaria para cumplir sus responsabilidades.

- En los hospitales analizados las metas y objetivos son conocidos por los jefes de servicio pero con frecuencia no son transmitidos a los equipos de trabajo. De la misma manera existe un gran desconocimiento de los resultados y una falta de retorno de la información de los resultados de obtenidos por los equipos a los miembros.

- La ausencia de indicadores y registros a nivel individual en el sector sanitario es una de las conclusiones más claras del estudio. Esta ausencia de indicadores hace muy difícil establecer un sistema de reconocimiento adecuado.

Luego de este trabajo podemos concluir que la efectividad de trabajo en equipo puede y deber medirse. El modelo IMO propuesto para representar los procesos que se desarrollan en el trabajo en equipo ha permitido asimismo, medir de manera cualitativa la efectividad del trabajo en equipo en los hospitales estudiados. Esta valoración/evaluación ha sido realizada a través del diseño, realización y posterior análisis de una entrevista construida para valorar en cada caso la existencia, importancia y nivel de aplicación de los factores implicados (y previamente definidos) en este modelo.

Se vislumbran como objetivos a futuro, por un lado, el poder evaluar el modelo en el conjunto de la red sanitaria para estos u otros equipos de trabajo. El disponer de conocimiento explícito acerca de 
Desarrollo metodológico para medir el trabajo en equipo

Viles, Elisabeth; Jaca, Carmen; Tanco, Martín; Medina, Allan

las fortalezas y las debilidades de su manera concreta de trabajar debiera de ser considerado una actividad con valor añadido en su camino a la mejora continua.

Por otro lado, el modelo podría ser adaptado para otros sectores industriales y/o de servicios, donde el trabajo en equipo constituya una manera habitual de trabajar.

\section{Referencias bibliográficas}

Borrill, Carol; West, Michael; Shapiro, David; Rees, Anne (2000). Team working and effectiveness in health care. British Journal of Health Care, Vol. 6, No. 8, Pp. 361-371.

Bower, Paul; Campbell, S. Stephen; Bojke, Chris; Sibbald, Bonnie (2003). Team structure, team climate and the quality of care in primary care: an observational study. Quality and Safety in Health Care, Vol. 12, No. 4, Pp. 273279.

Brannick, Michael; Salas, Eduardo; Prince, Carolyn (1997). Team performance assessment and measurement: Theory, methods, and applications. Lawerence Erlbaum Associates, Inc, Mahwah, New Jersey.

Cohen, Susan G.; Bailey, Diane E. (1997). What Makes Teams Work: Group Effectiveness Research from the Shop Floor to the Executive Suite. Journal of Management, Vol. 23, No. 3, Pp. 239-290.

Firth-Cozens Jenny (2004). Organizational Trust: The keystone to patient safety. Quality \& Safety in Health Care, Vol. 13, Pp. 56-61.

Guzzo, Richard A.; Dickinson, Marcus W. (1996).Teams in organizations: recent research on performance and effectiveness. Annual Review of Psychology, Vol. 47, Pp. 307-338.
Hackman, J. Richard (1990). Groups that work (and those that don't) : creating conditions for effective teamwork. Jossey-Bass, San Francisco.

Ilgen, Daniel R.; Hollenbeck, John R.; Johnson, Michael; Jundt, Dustin (2005). Team in organizations: From InputProcess-Output Models to IMOI Models. Annual Revision Psychologist, Vol. 56, Pp. 517-543.

Katzenbach, John R.; Smith, Douglas K. (1993). The Discipline of Teams. Harvard Business Review, Vol. 71, No. 2, Pp. 111-120.

Kozlowski, Steve W. J.; Ilgen, Daniel R. (2006). Enhancing the Effectiveness of Work Groups and Teams. Psychological Science in the Public Interest, Vol. 7, No. 3, Pp. 77-124.

Lemieux-Charles, Louise; McGuire, Wendy L. (2006). What Do We Know about Health Care Team Effectiveness? A Review of the Literature. Med. Care Res. Rev. Vol. 63, No. 3, Pp. 263-300.

Lemieux-Charles, Louise; Murray, Michael; Baker, G. Ross; Barnsley, Jan; Tasa, Kevin; Ibrahim, Salahadin A. (2002). The effects of quality improvement practices on team effectiveness: a mediational model. Journal of Organizational Behavior, Vol. 23, No. 5, Pp. 533-553.

Mantilla, Mercedes; Garcia, Denys (2010).Trabajo en equipos autodirigidos: competencias personales y conductas necesarias para su éxito. Revista Venezolana de Gerencia, Vol. 15, No. 49, Pp. 51-71.

Marks, Michelle A.; Mathieu, John E.; Zaccaro, Stephen J. (2001). A temporally based framework and taxonomy of team processes. The Academy of Management Review, Vol. 26, No. 3, Ada, Ohio [etc.], Pp. 356-376. 
Mathieu, John E.; Maynard, M. Travis; Rapp, Tammy; Gilson, Lucy (2008). Team Effectiveness 1997-2007: A Review of Recent Advancements and a Glimpse Into the Future. Journal of Management, Vol. 34, No. 3, Pp. 410-476.

McGrath, Joseph E.; Arrow, Holly; Berdahl, Jenny L. (2000). The study of groups: Past, present, and future, Erlbaum, Mahwah, New York.

Mendibil, Kepa; MacBryde, Jillian (2005). Designing effective team-based performance measurement systems: an integrated approach. No. 8.

Mickan Sharon; Rodger, Sylvia (2005). Effective Health Care Teams: A model of six characterisitcs developed from shared perceptions. Journal of Interprofessional Care, Vol. 19, No. 4, Pp. 358-370.

Mickan, Sharon; Rodger, Sylvia (2000). Characteristics of effective teams:a literature review. Australian Health Review, Vol. 23, No. 3.

Oandasan, Ivy; Baker, G. Ross; Barker, Keegan; Bosco, Carmela; D'Amour, Danielle; Jones, Linda; Kimpton, Shandra; Lemieux-Charles, Louise; Nasmith, Louise; San Martin Rodriguez, Leticia; Tepper, Joshua; Way, Daniel (2006). Teamwork in Healthcare: Promoting Effective Teamwork in Healthcare in Canada. Canadian Health Services Research Foundation, Ottawa.
Patton, Michael Quinn (2002). Qualitative Research and Evaluation Methods (3a. ed.). Thousand Oaks: Sage Publications Inc.

Rico, Ramón; Alcover de la Hera, Carlos María; Tabernero, Carmen (2010). Efectividad de los Equipos de Trabajo, una Revisión de la última década de investicación (1999-2009). Revista de Psicología del Trabajo y de las Organizaciones, Vol. 26, No. 1, Pp. 47-71.

Salas, Eduardo; Sims, Dana E.; Burke, C. Shawn (2005). Is there a "Big Five" in Teamwork? Small Group Research, Vol. 36, No. 5, Pp. 555-599.

Sundstrom, Eric; Mclntyre, Michael; Halfhill, Terry; Richards, Heather (2000). Work Groups: From the Hawthorne Studies to Work Teams of the 1990s and Beyond. Group Dynamics: Theory, Research and Practice. Vol. 4, No. 1, Pp. 44-67.

Thylefors, Ingela; Persson, Olle; Hellström, Daniel (2005). Team types, perceived efficiency and team climate in Swedish cross-professional teamwork. Journal of Interprofessional Care, Vol. 19, No. 2, Pp. 102-114.

Xyrichis, Andreas; Ream, Emma (2008). Teamwork: a concept analysis. Journal of advanced nursing, Vol. 61, No. 2, Pp. 232-241.

Zander Alvin (1994).Making groups effective. Jossey-Bass, San Francisco; Oxford. 